\title{
Die Universitäten liefern zu wenig Hausärzte!
}

\section{Die Kassenärztliche Bundesvereinigung warnt vor dramatischen Versorgungs- lücken: Der hausärztliche Nachwuchs bleibe aus. Schuld an der Misere seien die Universitäten. Die lassen die Kritik nicht auf sich sitzen.}

- Einerseits werden immer mehr Hausärzte gebraucht, anderseits gibt es immer weniger Nachwuchs. Die Kassenärztliche Bundesvereinigung (KBV) hat daher gefordert, die ärztliche Aus- und Weiterbildung an die Versorgungsnotwendigkeiten der Bevölkerung anzupassen. Vor allem müsse die ambulante Tätigkeit in der universitären Ausbildung zukünftig eine stärkere Rolle spielen.

\section{Positionspapier vorgestellt}

In einem Positionspapier der KBV-Vertreterversammlung heißt es, dass bis zum Jahr 2020 etwa 48000 niedergelassene Ärzte altersbedingt ausscheiden werden. Der Nachwuchs fehle jedoch: „Immer weniger junge Ärzte sind bereit, nach ihrer Ausbildung in der kurativen Patientenversorgung tätig zu werden“, heißt es in dem Papier.

Im Jahr 2012 haben der KBV zufolge 10127 Ärzte ihre Weiterbildung abgeschlossen. „Davon sind lediglich 949 Hausärzte“, sagte KBV-Vorstand Regina Feldmann anlässlich der Vorstellung des Papiers am Donnerstag in Berlin. Das entspreche 9,4\% aller Absolventen. Zum Vergleich: Im Jahr 2005 waren es noch 3506 Hausärzte. Die niedrige Anzahl der Absolventen deckt somit keinesfalls den steigenden Bedarf.

\section{Patienten häufiger ambulant behandelt}

Verschärft werde diese Entwicklung durch den steigenden Behandlungsbedarf einer älter werdenden Bevölkerung. Inzwischen könnten diese Patienten aufgrund des medizinischen Fortschritts oft in der Praxis statt in der Klinik be- handelt werden. „Die Zeit drängt. Wir müssen jetzt handeln“, warnte KBV-Vize Feldmann.

Der Hausarztmangel sei nur ein Teil des Problems. Künftig würden fast alle Grundversorger fehlen: Auch bei Augen-, Haut-, und Hals-Nasen-OhrenÄrzten fehle der Nachwuchs. Allerdings reagierten Universitäten bislang noch nicht auf diesen negativen Trend.

\section{Der Wunsch, Hausarzt zu werden, sinkt während des Studiums}

Nach einer Studie der Universität Trier können sich am Anfang des Studiums noch $40 \%$ der Studenten vorstellen, als Hausarzt tätig zu werden. Am Ende des Studiums sind es nur noch $10 \%$. Die ärztliche Ausbildung sei somit nicht mehr zeitgemäß - weder inhaltlich noch strukturell, kritisierte Feldmann. Sie bilde in keiner Weise die Versorgungsrealität ab. Den Stellenwert der ambulanten Medizin

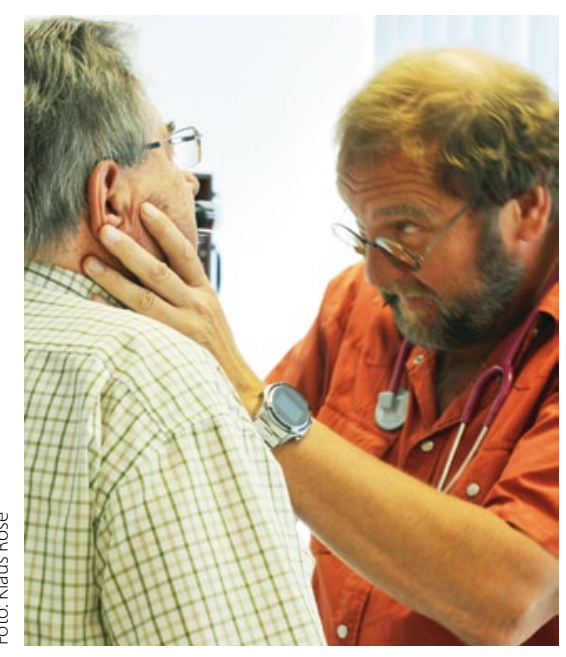

Ist der breitbandig aufgestellte Hausarzt wirklich ein Auslaufmodell? könne in den universitären Ausbildungseinrichtungen bestenfalls erahnt werden. An 36 medizinischen Hochschulen werden etwa 0,5\% aller Patienten versorgt, mit einer durchschnittlichen Liegezeit von sieben Tagen.

\section{Pflichtweiterbildung ausbauen}

Auch bei der Weiterbildung der Ärzte sieht Feldmann Handlungsbedarf. Hier solle eine mindestens sechsmonatige Pflichtweiterbildung über das Fach Allgemeinmedizin hinaus auf weitere patientennahe Gebiete im ambulanten Bereich verankert werden. „Organisatorisch kann dies über die bestehenden Koordinierungsstellen erfolgen, die derzeit schon dem Arzt in Weiterbildung als Anlaufstelle dienen und ausgebaut werden könnten“, heißt es in dem Papier der KBV-VV.

\section{Fakultätentag widerspricht Schuldzuweisungen}

Der Medizinische Fakultätentag (MFT) weist die Kritik an der universitären Ausbildung zurück. „Die Schuldzuweisungen sind unangemessen, der Wunsch, als Allgemeinmediziner zu arbeiten ist in den vergangenen Jahren gestiegen“, erklärte Dr. Volker Hildebrandt, Generalsekretär des MFT.

„Allerdings wollen viele als Angestellte arbeiten und fürchten sich vor der finanziellen Belastung einer Praxis." Er betont, dass in einer Umfrage unter Studenten im Jahr 2010 deutlich wurde, dass die Allgemeinmedizin auf Platz zwei hinter der Inneren Medizin rangiere. Die Ergebnisse der Umfrage wurden damals gemeinsam von KBV und MFT erhoben.

SunNa Gieseke, RebecCA BeERHEIDE - 